\title{
Newborn care practices and home-based postnatal newborn care programme - Mewat, Haryana, India, 2013
}

\author{
Latika Nath Sinha, a Prabhdeep Kaur, a Rakesh Gupta, ${ }^{b}$ Suresh Dalpath, ${ }^{c}$ Vinod Goyalc and Manoj Murhekara \\ Correspondence to Latika Nath (e-mail: drlatika@gmail.com).
}

Background: In India, the Home Based Postnatal Newborn Care programme by Accredited Social Health Activists (ASHAs) under the National Rural Health Mission was initiated in 2011 to reduce neonatal mortality rates (NMRs). ASHAs get cash incentives for six postnatal home visits for newborn care. We studied newborn care practices among mothers in Mewat, Haryana, having a high NMR and determined risk factors for unsafe practices and described the knowledge and skills of ASHAs during home visits.

Methods: A cross-sectional survey was conducted among mothers who had delivered a child during the previous seven months using cluster sampling. We interviewed mothers and ASHAs in the selected subcentres using semi-structured questionnaires on the six safe newborn care practices, namely safe breastfeeding, keeping cord and eyes clean, wrapping baby, kangaroo care, delayed bathing and hand washing.

Results: We interviewed 320 mothers, 61 ASHAs and observed 19 home visits. Overall, 60\% of mothers adopted less than three safe practices. Wrapping newborns (96\%) and delayed bathing (64\%) were better adopted than cord care $(49 \%)$, safe breastfeeding (48\%), hand washing (30\%), kangaroo care $(20 \%)$ and eye care $(9 \%)$. Cultural beliefs and traditional birth attendants influenced the mother's practices. The lack of supervision by auxiliary nurse midwives (ANM), delayed referral and transportation were the other challenges.

Conclusion: Knowledge-practice gaps existed among mothers counselled by ASHAs. Poor utilization of reproductive and child health services decreased opportunities for ASHA-mother dialogue on safe practices. Recommendations included training ANMs, training TBAs as ASHAs, innovative communication strategies for ASHAs and improved referral system.

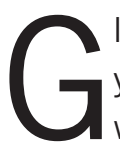
lobally, over 130 million babies are born every year, and almost 4 million die in the first four weeks of life. ${ }^{1}$ Presently, the infant mortality rate (IMR) for India is 47 per 1000 live births, and the neonatal mortality rate (NMR) is 32 per 1000 live births. ${ }^{2}$ India aims for a two-thirds reduction in IMR, from the 1990 level of $84 / 1000$ live births to $28 / 1000$ live births by $2015 .^{3}$ The NMR contributes to $68 \%$ of the IMR, and any further reduction in IMR can only come from a decline in NMR. ${ }^{4}$ The effective interventions to reduce the NMR component of the IMR in settings with high mortality and weak health systems include outreach, family-community care, health education to improve home-care practices and a simultaneous expansion of clinical care. ${ }^{5}$ Several trials incorporating communitylevel interventions in South Asia (Pakistan, Bangladesh, Nepal) and sub-Saharan Africa (Nigeria, Malawi, Ethiopia) have shown reductions in neonatal mortality rates. ${ }^{6}$ Community-based trials from Maharashtra and Uttar Pradesh in India showed $62 \%$ and $54 \%$ reductions in neonatal mortality, respectively, through multiple prenatal and postnatal home visits by trained community level health workers. ${ }^{7,8}$ Based on evidence from these trials, and WHO and UNICEF recommendations, the Government of India introduced home-based newborn care involving community-based workers, Accredited Social Health Activists (ASHAs), under the National Rural Health Mission in June 2011. ${ }^{4}$

Mewat district in Haryana has the highest IMR of $91 / 1000$ live births and NMR of $47 / 1000$ live births in the state. ${ }^{9,10}$ The Home Based Post Natal Newborn Care (HBPNC) programme was implemented with technical assistance from the United Nations Office for Project Services-Norway India Partnership Initiative in addition to health system strengthening in Mewat. ${ }^{11,12}$ An ASHA

\footnotetext{
National Institute of Epidemiology, Chennai, India.

National Rural Health Mission, Haryana, India.

Child Health and Immunization, Directorate of Health Services, Haryana, India. Submitted: 31 January 2014; Published: 29 September 2014

doi: 10.5365/wpsar.2014.5.1.006
} 
is paid a monetary incentive for making one prenatal visit (in eighth month of pregnancy) and six postnatal (on days $1,3,7,14,28$ and 42 ) home visits. ${ }^{4}$

State programme managers assessed the level of implementation of various interventions targeted to improve newborn care at the community level; mothers were usually dependant on traditional birth attendants (TBAs) who conducted home deliveries and subsequently advised them on newborn care. Therefore, we estimated the knowledge, attitude and practices among mothers regarding newborn care and determined the factors associated with unsafe neonatal care practices by mothers. We also estimated key indicators of the HBPNC programme for training, knowledge, timeliness, quality and documentation of ASHAs' home visits.

\section{METHODS}

\section{Study area}

Mewat district in the south of Haryana has a population of $1089406 .{ }^{13}$ It has six rural blocks with 431 villages. Muslims comprise $82 \%$ of the population, and the majority of the people in the rural part of the district are farmers. The female literacy rate in the district is $36.6 \% .^{14}$ In an otherwise well-performing state this district is a high focus area due to its poor heath indicators and infrastructure. ${ }^{15,16}$ Of the 715 ASHAs in the district providing newborn care, 438 had received two rounds of training on providing home-based care.

\section{Study population and study design}

We surveyed mothers who had delivered a live child within the reference period (1 July 2012 to 31 January 2013) and who resided in the same cluster health subcentre (health unit catering to a population of 5000 with an auxiliary nurse midwife [ANM] in charge) during delivery and during the data-collection period. The corresponding ASHAs in the subcentre areas were also included in the study. It was a cross-sectional study.

\section{Sampling procedure and sample size}

We used cluster sampling techniques with subcentres as Primary Sampling Units. The estimated number of pregnancies in one year is 35000 (birth rate is $35 / 1000$ live births); per the District Level Household and Facility Survey 3, 29\% of mothers practised safe breastfeeding practices in Mewat. ${ }^{17}$ A relative precision of $20 \%$ (equivalent to an absolute precision of $5.8 \%$ on either side) was used and was rounded off to $6 \%$ to calculate sample size. Assuming the same $29 \%$ of mothers knew other safe newborn care practices, a 95\% confidence interval, an absolute precision of $\pm 6 \%$ and design effect of 1.38 , a sample size of 320 mothers was needed. We interviewed 20 mothers in each of the 16 clusters. We selected the clusters using probability-proportionate-tosize linear systematic sampling method. Mothers within the selected cluster were selected using the line list of deliveries within the reference period in the subcentre. A 5\% non-response rate was seen among the mothers. The first respondent was selected randomly from the list, while the subsequent mothers were selected from the chronological order of deliveries, giving priority to more recent deliveries. All the ASHAs serving the subcentre areas were also interviewed.

\section{Data collection}

We used a questionnaire to collect data from mothers regarding socio-demographic characteristics; knowledge, attitude and practices of newborn care and their interaction with ASHAs during the course of their last pregnancy up to 42 days after delivery. ASHAs were interviewed regarding their knowledge; practices; important skills such as taking weight, temperature and recognizing danger signs; motivating factors and hindrances in providing home-based newborn care. ASHAs' activities during home visits were observed using a checklist. Data were collected by trained field investigators and the principal investigator between January and March 2013. Operational definitions are shown in Box 1.

\section{Data analysis}

Data were entered and analysed using Epi Info (version 3.5.3) and MS Excel software. We estimated the proportion for awareness, practice of safe newborn care and reasons for not adopting safe practices among mothers. We also estimated the proportion of ASHAs giving correct advice on safe practices, training, timeliness, quality and documentation of home visits.

\section{Human subject protection}

The study was approved by the Institutional Ethics Committee of the National Institute of Epidemiology, 


\section{Box 1. Operations definitions}

Mother: Mothers of babies delivered within the period 1 July 2012 to 31 January 2013.

Neonate/Newborn: Childhood period from zero to 28 days. Early breastfeeding: A mother putting her newborn child to breast within one to six hours of delivery.

Colostrum feeding: A mother feeding her newborn the first milk (colostrum) secreted from her breast after delivery.

Exclusive breastfeeding: A mother who fed her newborn only breast milk for the entire neonatal period.

Safe breastfeeding: A mother who adopted all three of the breastfeeding practices.

Keeping cord stump and eyes clean: Not applying anything to the umbilical cord of the neonate in the entire neonatal period except on medical advice by a qualified doctor.

Not applying anything to the eyes except on medical advice.

Wrapping baby: Wrapping the neonate in multiple layers of clothing.

Kangaroo care (skin-to-skin care): Holding the neonate with his/her bare skin in contact with the bare skin of mother/ caregiver.

Delaying bath: Not giving a bath to the newborn until at least 48 hours after delivery.

Hand washing: Washing hands by mother/caregiver with soap and water each time before handling the child.

Safe newborn care practice: A mother adopting the following six practices - safe breastfeeding, keeping cord clean and eye care, wrapping baby, kangaroo care, delaying bath and hand washing.

ASHA: A woman of a village (married/widowed/divorced) 25-45 years of age recruited as a link worker between community and health delivery systems under the National Rural Health Mission on a population of 1000.

"102 ambulance referral service" (Haryana Swasthya 102 Vaahan Sewa): Ambulance service provided by the government in response to a call on a toll-free number ("102") in medical emergencies.

Chennai. We briefed the participants about the study, gave them an information sheet and took written informed consent from them. Special care was taken to explain the study to illiterate mothers before their consent was taken in the presence of witnesses.

\section{RESULTS}

\section{Socio demographic characteristics}

We interviewed 320 mothers, 61 ASHAs and directly observed home visits of 19 ASHAs. Most mothers were housewives $(211,66 \%)$, illiterate $(243,76 \%)$ and Muslims (226, 71\%). Also, 118 (37\%) did not have any antenatal check-up during their pregnancy, and $124(39 \%)$ had no antenatal check-up in the first trimester. Home deliveries by traditional birth attendants (TBAs) were reported by 165 (52\%) mothers, and
$63(36 \%)$ mothers had not been counselled on newborn care. Among the 155 hospital deliveries, 94\% had a hospital stay of less than 48 hours post-delivery. One hundred and sixty-two (51\%) mothers had three or more children, and the majority $(275,86 \%)$ had less than two years spacing between children.

\section{Safe practices}

Overall, 237 (74\%) mothers started breastfeeding within the first hour, 279 (87\%) fed colostrum, and $188(58 \%)$ mothers exclusively breastfed their newborn. The baby was wrapped in multilayers by $308(96 \%)$ mothers, and $64(20 \%)$ of them practised the kangaroo care method. Delayed bath after 48 hours was given to babies by 205 (64\%) mothers. Half of the mothers (158, 49\%) did not apply anything on the cord stump, and $28(9 \%)$ mothers kept the eyes clean. The proportion of mothers aware of, yet not practising, was highest for hand washing with soap and water before handling a newborn $(45 \%)$, cord care $(42 \%)$ and exclusive breastfeeding (32\%) (Figure 1).

The common reasons stated by mothers for nonadoption of safe practices were that they prefer to follow tradition, advice/influence of local TBAs (midwives), family pressure and personal choice besides medical reasons. TBAs bathed the newborns immediately, used unsterile thread to tie the cord and advised mothers to coat the newborns' cord stumps with multiple applications. Among the mothers who were aware of, yet not following safe practices, the TBA influence was reported as the leading cause by $37 \%$ of the mothers for the practice of delayed breastfeeding, $26 \%$ for not keeping the cord clean and by $17 \%$ for bathing immediately after birth. For hand washing with soap and water, $59 \%$ of mothers stated they "did not consider it necessary," and 15\% respondents said they had "no time" for this practice (Figure 2).

\section{Risk factors for unsafe practices}

\section{ASHAs' knowledge and service delivery}

Among the ASHAs, 51 (83.6\%) knew how to use the weighing scale, and $43(70 \%)$ could correctly take a temperature using a digital thermometer. Although 90\% of the ASHAs interviewed knew the importance of most of the safe practices, the lesser known and advocated safe practices were delayed bathing by $52(85.2 \%)$ 
Figure 1. Awareness and adoption of newborn care practices among mothers in Mewat, Haryana, India, $2013(n=320)$

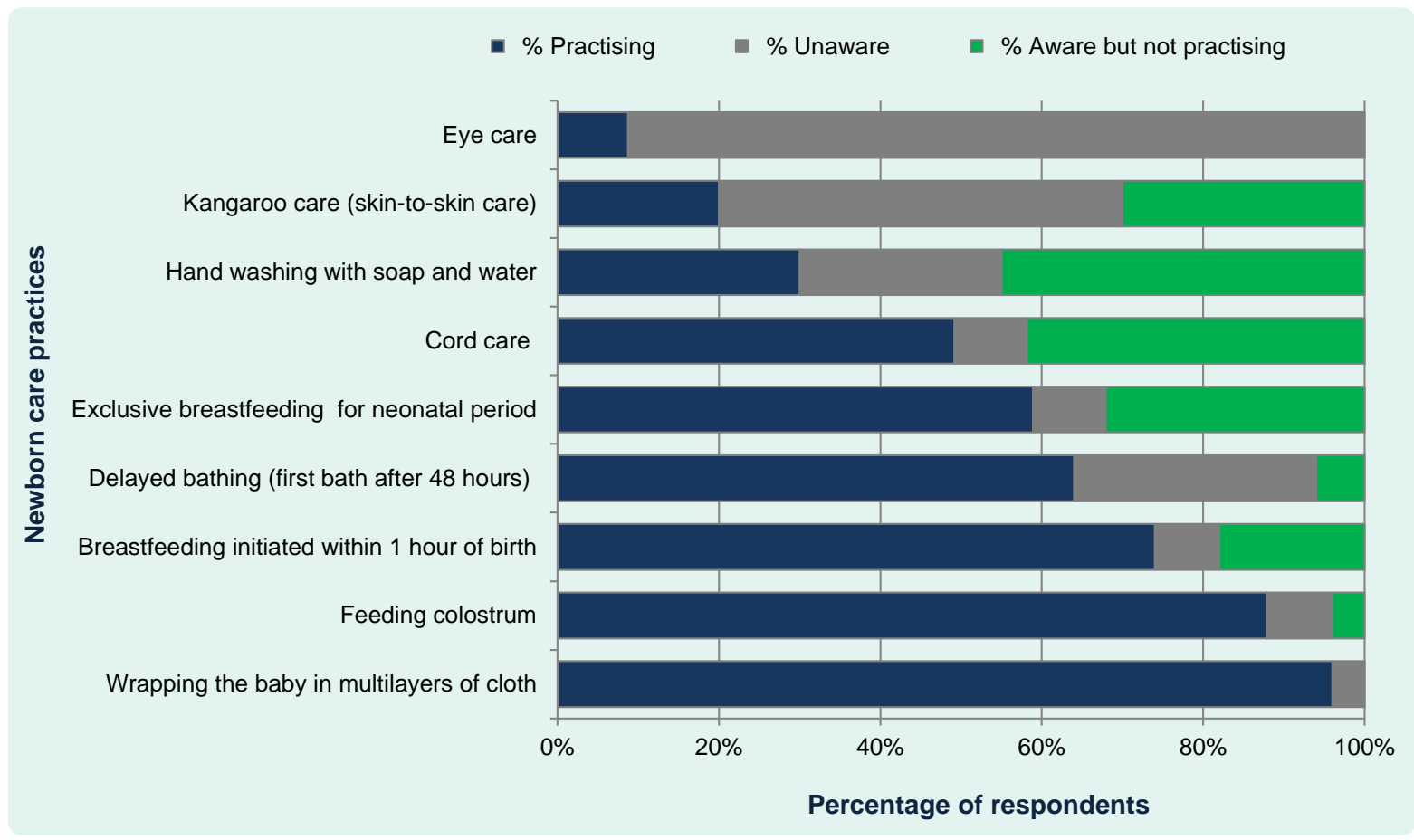

Figure 2. Reasons given by mothers for not practising safe newborn care by each practice, Mewat, Haryana, India, 2013

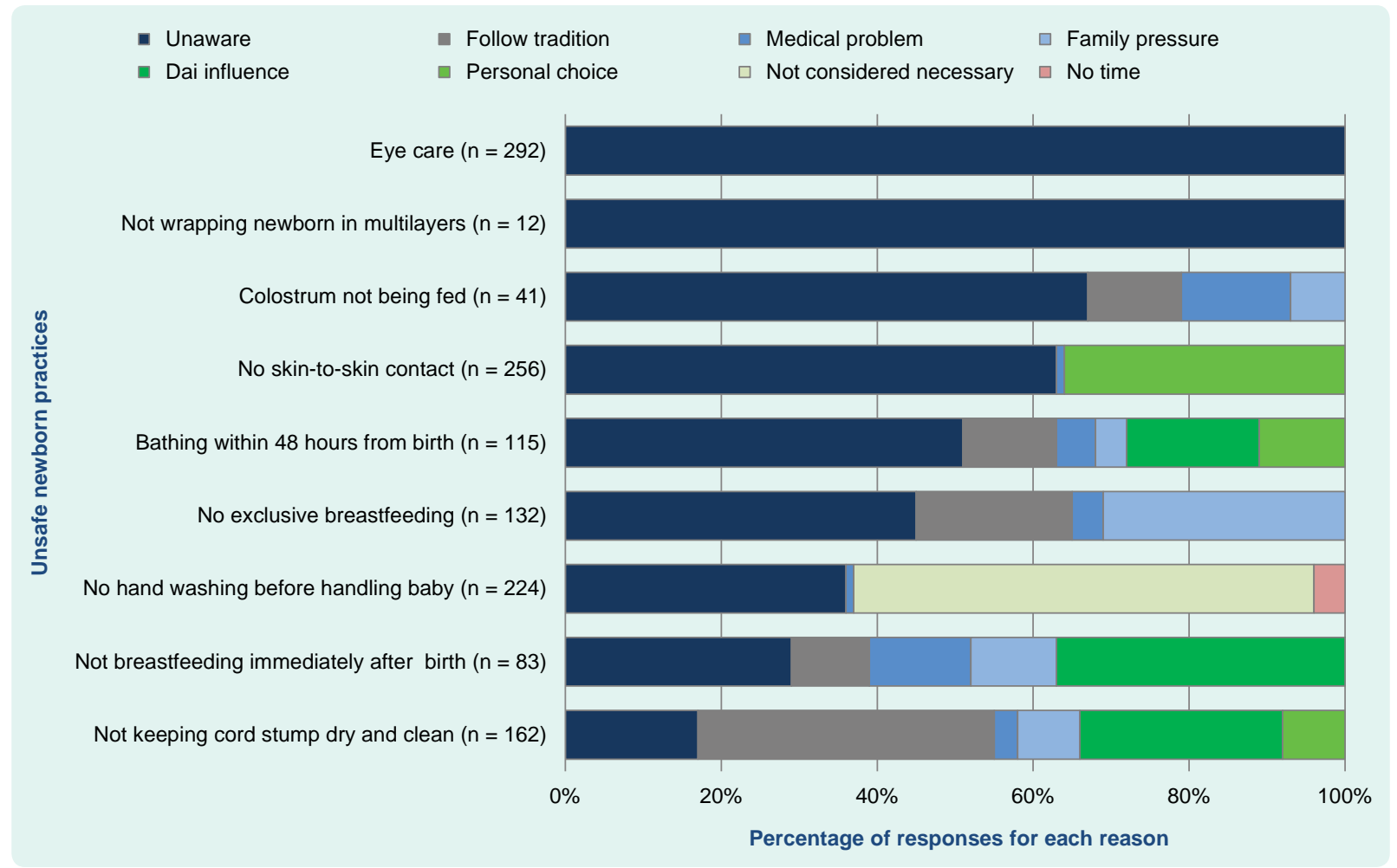

ASHAs, kangaroo care method by $44(72 \%)$, hand one of the birth (among home deliveries), and $15 \%$ washing with soap and water before handling newborn by of the mothers recalled all seven visits by an ASHA. $41(67 \%)$ and safe eye care by 24 (39\%) of the ASHAs. On direct observation of 19 ASHAs, only $32 \%$ washed Only $30 \%$ of the mothers reported ASHAs' visits on day their hands before handling the baby, $40 \%$ gave 
Table 1. Key activities of Home Based Newborn Care by trained ASHAs in Mewat, Haryana, India, 2013

\begin{tabular}{|c|c|c|c|}
\hline Key activities & $n$ & $\%$ & Total \\
\hline \multicolumn{4}{|l|}{ Timeliness of home visits } \\
\hline Mothers who reported ASHAs' visit on first day (within 24 hours of home delivery) & 55 & 33 & 165 \\
\hline Mothers who reported all seven postnatal home visits by ASHAs for newborn care & 47 & 15 & 320 \\
\hline \multicolumn{4}{|l|}{ Quality of visit (by direct observation of 19 home visits) } \\
\hline Carrying weighing scale & 14 & 74 & 19 \\
\hline Carrying thermometer & 11 & 58 & 19 \\
\hline Took weight of newborn correctly after adjusting for zero error & 10 & 71 & 14 \\
\hline Took temperature of newborn correctly & 8 & 73 & 11 \\
\hline Gave information on immunization (BCG, polio, hepatitis B) & 18 & 95 & 19 \\
\hline Gave information on birth registration to mother & 17 & 90 & 19 \\
\hline Advised on cord care & 16 & 84 & 19 \\
\hline Physically examined newborn for jaundice/rash & 14 & 74 & 19 \\
\hline Advised on kangaroo care & 8 & 42 & 19 \\
\hline Gave information about 102 ambulance service for sick newborn & 7 & 37 & 19 \\
\hline Advised on recognition of danger signs & 7 & 37 & 19 \\
\hline Washed hands before handling newborn & 6 & 32 & 19 \\
\hline Advised on hand washing before handling newborn & 4 & 21 & 19 \\
\hline Advised on eye care & 3 & 16 & 19 \\
\hline Used the pictorial flip chart to explain safe practices to mothers & 0 & 0 & 19 \\
\hline \multicolumn{4}{|l|}{ Documentation } \\
\hline ASHAs filling out the postnatal care cards & 11 & 58 & 19 \\
\hline ASHAs found with erroneously filled out postnatal care cards & 38 & 62 & 61 \\
\hline ASHAs visits crosschecked/verified by ANMs & 4 & 7 & 61 \\
\hline \multicolumn{4}{|l|}{ Status of referrals made: } \\
\hline Number of ASHAs who referred sick newborns in last seven months & 34 & 56 & 61 \\
\hline Availability of 102 ambulance for these referrals & 8 & 24 & 34 \\
\hline Number of ASHAs who reported delays/refusals in 102 service & 26 & 43 & 61 \\
\hline
\end{tabular}

information about 102 ambulance referral service (Box 1) and 37\% mentioned danger signs. None of the ASHAs used the pictorial flip chart given to them in their trainings to increase the mothers' understanding. There was no monitoring or supervision of the ASHAs' home visits by the ANMs. Among the ASHAs, 58\% were simultaneously filling out the postnatal cards. Delay in 102 service was reported by $43 \%$ of the ASHAs (Table 1).

We analysed the dose response relationship to determine the relationship of each of the unsafe practices with the decrease in the number of post-delivery home visits by ASHAs. There was a significant increasing trend of unsafe newborn care practices with regards to early bathing and cord care with fewer visits $(P<0.01)$ but not in early breastfeeding and kangaroo care (Table 2).

\section{DISCUSSION}

Our study showed adoption of select newborn care practices among mothers; however, there were gaps in the adoption of a few practices either due to lack of awareness or influence of other stakeholders. ASHAs played an important role in influencing the mother's behaviour; however, lack of utilization of various Reproductive and Child Health ( $\mathrm{RCH}$ ) programme services limited the opportunities for contact with the health system.

Improvement in adoption of breastfeeding practices, except exclusive breastfeeding, can possibly be attributed to the consistent efforts made by the ASHAs to educate mothers during home visits. A similar change was seen in a study in Sri Lanka on involvement 
Table 2. Unsafe newborn care practices versus number of ASHA visits among mothers in Mewat, Haryana, India, $2013(n=320)$

\begin{tabular}{|c|c|c|c|c|c|c|c|}
\hline \multicolumn{8}{|c|}{ Number of home visits } \\
\hline \multicolumn{2}{|l|}{ Unsafe practices } & $>7$ visits & $5-6$ visits & $3-4$ visits & $0-2$ visits & $X^{2}$ for trend & $p$-value \\
\hline \multirow[t]{2}{*}{ Bathing baby before 48 hours } & $\%$ & 12 & 24.0 & 25.0 & 38.0 & & \\
\hline & OR & 1 & 2.4 & 2.2 & 3.8 & 12.1 & $<0.01$ \\
\hline \multirow[t]{2}{*}{ Unclean cord } & $\%$ & 18 & 23.0 & 32.0 & 28.0 & & \\
\hline & OR & 1 & 1.4 & 2.3 & 2.4 & 8.9 & $<0.01$ \\
\hline \multirow[t]{2}{*}{ No exclusive breastfeeding } & $\%$ & 18 & 26.0 & 25.0 & 31.0 & & \\
\hline & OR & 1 & 1.6 & 1.3 & 2.5 & 5.9 & $<0.05$ \\
\hline \multirow[t]{2}{*}{ Not feeding colostrum } & $\%$ & 10 & 24.0 & 32.0 & 34.0 & & \\
\hline & OR & 1 & 2.6 & 3.2 & 4.2 & 5.7 & $<0.05$ \\
\hline \multirow[t]{2}{*}{ No hand washing } & $\%$ & 23 & 23.0 & 26.0 & 29.0 & & \\
\hline & OR & 1 & 0.9 & 1.0 & 2.7 & 5.4 & $<0.05$ \\
\hline \multirow[t]{2}{*}{ No early breastfeeding } & $\%$ & 20 & 21.0 & 29.0 & 30.0 & & \\
\hline & OR & 1 & 0.9 & 1.3 & 1.7 & 2.8 & NS \\
\hline \multirow[t]{2}{*}{ No kangaroo care } & $\%$ & 60 & 75.0 & 66.0 & 55.0 & & \\
\hline & OR & 1 & 1.8 & 2.5 & 1.5 & 1.5 & NS \\
\hline \multirow[t]{2}{*}{ All practices } & $\%$ & 21 & 25.0 & 26.0 & 29.0 & & \\
\hline & OR & 1 & 1.3 & 1.4 & 2.5 & 5.8 & $<0.05$ \\
\hline
\end{tabular}

OR, odds ratio; NS, not significant

of health workers in postnatal home visits. ${ }^{18}$ The odds of practising safe breastfeeding among mothers was found to be higher (odds ratio: 7.6 ; $95 \%$ confidence interval: 6.03-9.71) among those who received one-on-one counselling and hands-on support in an intervention trial where community-based trained workers made home visits within the first three days of birth in Bangladesh. ${ }^{19}$ Therefore, more intensive counselling by ASHAs in the first three postnatal visits might further improve adoption of this practice.

Cord care and hand washing were the two practices that were not adopted despite awareness. Hand washing was primarily influenced by low risk perception by mother and cord care was influenced by TBAs who conducted the home delivery. Similar low coverage of clean cord care was seen in Southern Nepal among home deliveries, increasing the risk of umbilical infections by $29 \%$ (due to topical applications) and $62 \%$ (due to other unclean practices). ${ }^{17} \mathrm{~A}$ randomized controlled trial in Pakistan showed that training TBAs and their integration into the health system to propagate newer safe practices, such as cord care, were effective in reducing neonatal mortality by $30 \% .{ }^{20}$ We need to consider strategies to better engage the TBAs to increase the adoption of these practices.
Low awareness regarding kangaroo care and eye care led to low adoption of these practices. ASHAs were not emphasizing the multiple benefits of kangaroo care such as prevention of hypothermia and the role of skin-toskin contact to promote longer duration of breastfeeding, increased bonding, reduced pain responses and crying in newborns. ${ }^{21,22}$ Adoption of these newer practices requires engaging not only the mothers but also the community at large using innovative communication strategies like messages, songs, flip charts and sharing of personal experiences as done in the Shivgarh intervention trial. ${ }^{23}$

There was low utilization of $\mathrm{RCH}$ services in Mewat for family planning, antenatal care and delivery. This reduced the number of contacts of the mother with ASHA/ ANM for counselling on birth preparedness, benefits of institutional deliveries and postnatal newborn care. ${ }^{6}$ The lack of acceptance of family planning may lead to poor birth spacing in this population that increases the risk of pre-term and low birth weight babies who are more susceptible to infections and hence mortality. ${ }^{24,25}$ An ASHA visit on the first day after home delivery might influence the mother, but less than half of the newborns were visited on day one in our study. The importance of a first day home visit in reducing mortality by $67 \%$ was highlighted in a meta-analysis. ${ }^{3}$ Visits on subsequent 
days by ASHAs are important for early recognition of feeding problems and treatable infections followed by prompt referrals to reduce morbidity and mortality in newborns. ${ }^{3}$ One of the key issues emerging from our study was poor supervision of ASHAs' home visits by ANM. Observation revealed that inadequate documentation and inability to use instruments was noticeable among illiterate ASHAs who were formerly TBAs. In addition, lack of ANM training on HBPNC guidelines might be preventing effective supervision of ASHA visits and their documentation.

We have not included mothers who went to their maternal/native place outside the subcentre areas for their delivery in this study. One of our other limitations was a problem in recall by mothers of ASHA visits even though the reference period was seven months.

Our sample was representative of the district, and the results can be extrapolated to the entire district. This study has shown good implementation and outcomes in an underdeveloped district; therefore, the benefits of this programme might be similar in other districts with even better health systems.

In conclusion, mothers adopted a few safe practices; however, there were gaps in the adoption of several safe practices despite being informed of them. ASHAs seem to have played a key role in facilitating the adoption of safe practices; however, the quality of services can be further improved. There was a need for innovative training strategies to improve the ASHAs' skills and a need for engaging communities including elder family members and TBAs in counselling sessions to increase community acceptance of safe practices. Practices such as kangaroo care and hand washing need more emphasis in the training. Strengthening delivery of $\mathrm{RCH}$ services would increase opportunities for ASHA-mother dialogue on safe practices. Training of TBAs and recruiting them as ASHAs would further strengthen the programme. Training of all ANMs in HBPNC guidelines to enable supportive supervision in the field was recommended. The responsiveness of the 102 ambulance services to newborn emergencies also needed improvement.

The local health authorities implemented some of our study recommendations immediately. Approximately 75 TBAs were inducted into the ASHA system, and a cadre of ASHA facilitators were trained for better supervision of home visits. There was re-training of ASHAs to enhance communication skills. A grievance unit was established to address delays in referral transport. We need followup studies to determine the effectiveness of the actions taken by the health authorities.

\section{Conflicts of interest}

None declared.

Funding

The funding was done by the National Rural Health Mission, Haryana, India.

Note:

This paper was accepted for publication as a result of a call for articles to all presenters at the 2013 TEPHINET conference.

\section{References:}

1. Lawn JE, Cousens S, Zupan J; Lancet Neonatal Survival Steering Team. 4 million neonatal deaths: When? Where? Why? Lancet, 2005, 365:891-900. doi:10.1016/S0140-6736(05)71048-5 pmid: 15752534

2. World Bank report on Neonatal Mortality Rates. Washington, DC, World Bank, 2012.

3. Gogia S Sr et al. Community based newborn care: a systematic review and metaanalysis of evidence: UNICEF-PHFI series on newborn and child health, India. Indian Pediatrics, 2011, 48:537546. doi:10.1007/s13312-011-0096-8 pmid:21813923

4. Home Based Newborn Care-Operational Guildelines. New Delhi, Ministry of Health and Family Welfare, 2011, 8-10 (http://www.nihfw.org/Doc/NCHRC-Publications/Operational Guidelines on Home Based Newborn Care (HBNC).pdf, accessed 25 August 2014).

5. Darmstadt GL et al.; Lancet Neonatal Survival Steering Team. Evidence-based, cost-effective interventions: how many newborn babies can we save? Lancet, 2005, 365:977-988. doi:10.1016/ S0140-6736(05)71088-6 pmid:15767001

6. Nair $\mathrm{N}$ et al. Improving newborn survival in low-income countries: community-based approaches and lessons from South Asia. PLoS Medicine, 2010, 7:e1000246. doi:10.1371/journal. pmed.1000246 pmid:20386728

7. Kumar $\vee$ et al.; Saksham Study Group. Effect of communitybased behaviour change management on neonatal mortality in Shivgarh, Uttar Pradesh, India: a cluster-randomised controlled trial. Lancet, 2008, 372:1151-1162. doi:10.1016/S01406736(08)61483-X pmid:18926277

8. Bang AT et al. Neonatal and infant mortality in the ten years (1993 to 2003) of the Gadchiroli field trial: effect of home-based neonatal care. Journal of Perinatology, 2005, 25 Suppl 1;S92107. doi:10.1038/sj.jp.7211277 pmid:15791283

9. National Health System Resource Centre. 5th Common Review Mission Report 2011. Haryana. New Delhi, Ministry of Health and Family Welfare, 2012 (http://mohfw.nic.in/WriteReadData/ 
I892s/3174333004Main\%20Report 5th_CRM\%20.pdf, accessed 25 August 2014).

10. Lahariya $C$ et al.; INCLEN Program Evaluation Network International (IPEN). Interdistrict variations in child health status and health services utilization: lessons for health sector priority setting and planning from a cross-sectional survey in rural India. The National Medical Journal of India, 2012, 25:137-141. pmid:22963289

11. 12th Norway India Partnership Initiative Programme management group-minutes of meetings. New Delhi, Norway India Partnership Initiative, 2011.

12. Annual Report 2011: Norway India Partnership Initiative. New Delhi, Norway India Partnership Initiative, 2011.

13. Census India 2011. New Delhi, Ministry of Home Affairs, 2011.

14. Ministry of Minority Affairs and Indian Council of Social Science Research. A baseline survey of minority concentration districts of India Mewat (Haryana). New Delhi, Institute for Human Development, 2008 (http://www.icssr.org/Mewat[1].pdf, accessed 25 August 2014).

15. National Rural Health Mission. Strategy Initiative to catch up with MDG Goals 4 and 5 - Accelerating Strategy for mother and child survival in high focus states and high focus districts in non high focus states. New Delhi, Ministry of Health and Family Welfare, 2009.

16. National Rural Health Mission for the Twelfth Five-Year Plan. New Delhi, Ministry of Health and Family Welfare, 2012.

17. Mullany LC et al. Risk factors for umbilical cord infection among newborns of southern Nepal. The American Journal of Epidemiology, 2007, 165:203-211. doi:10.1093/aje/kwj356 pmid: 17065275
18. Senarath U, Fernando DN, Rodrigo I. Newborn care practices at home: effect of a hospital-based intervention in Sri Lanka. Journal of Tropical Pediatrics, 2007, 53:113-118. doi:10.1093/tropej/ fml080 pmid:17169975

19. Mannan I Sr et al.; Bangladesh Projahnmo Study Group. Can early postpartum home visits by trained community health workers improve breastfeeding of newborns? Journal of Perinatology, 2008, 28:632-640. doi:10.1038/jp.2008.64 pmid:18596714

20. Khadduri $R$ et al. Household knowledge and practices of newborn and maternal health in Haripur district, Pakistan. Journal of Perinatology, 2008, 28:182-187. doi:10.1038/sj.jp.7211903 pmid:18059464

21. Johnston CC et al. Kangaroo care is effective in diminishing pain response in preterm neonates. Archives of Pediatrics \& Adolescent Medicine, 2003, 157:1084-1088. doi:10.1001/ archpedi.157.11.1084 pmid:14609899

22. Conde-Agudelo A, Belizán JM, Diaz-Rossello J. Kangaroo mother care to reduce morbidity and mortality in low birthweight infants. The Cochrane Database of Systematic Reviews, 2011 , (3):CD002771. pmid:21412879

23. USAID-ACCESS. Demystifying community mobilization: an effective strategy to improve maternal and newborn health. Washington, DC, United States Agency for International Development, 2007 (http://pdf.usaid.gov/pdf_docs/PNADI338. pdf, accessed 8 September 2014).

24. Joshi HS et al. Risk Factors Associated with Low Birth Weight in Newborns. Indian Journal of Community Medicine, 2005, 30:142-143.

25. Haaga JG. Mechanisms for the association of maternal age, parity, birth spacing with infant health. California, RAND Labor and Population, 1991 (http://www.rand.org/pubs/notes/N2991.html, accessed 25 August 2014). 